\title{
Clustering of Beijing genotype Mycobacterium tuberculosis isolates from the Mekong delta in Vietnam on the basis of variable number of tandem repeat versus restriction fragment length polymorphism typing
}

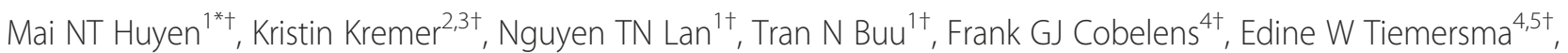
Petra de $\mathrm{Haas}^{3+}$ and Dick van Soolingen ${ }^{3,6+}$

\begin{abstract}
Background: In comparison to restriction fragment length polymorphism (RFLP) typing, variable number of tandem repeat (VNTR) typing is easier to perform, faster and yields results in a simple, numerical format. Therefore, this technique has gained recognition as the new international gold standard in typing of Mycobacterium tuberculosis. However, some reports indicated that VNTR typing may be less suitable for Beijing genotype isolates. We therefore compared the performance of internationally standardized RFLP and 24 loci VNTR typing to discriminate among 100 Beijing genotype isolates from the Southern Vietnam.
\end{abstract}

Methods: Hundred Beijing genotype strains defined by spoligotyping were randomly selected and typed by RFLP and VNTR typing. The discriminatory power of VNTR and RFLP typing was compared using the Bionumerics software.

Results: Among 95 Beijing strains available for analysis, 14 clusters were identified comprising 34 strains and 61 unique profiles in 24 loci VNTR typing ((Hunter Gaston Discrimination Index (HGDI = 0.994)). 13 clusters containing 31 strains and 64 unique patterns in RFLP typing ( $\mathrm{HGDI}=0.994)$ were found. Nine RFLP clusters were subdivided by VNTR typing and 12 VNTR clusters were split by RFLP. Five isolates (5\%) revealing double alleles or no signal in two or more loci in VNTR typing could not be analyzed.

Conclusions: Overall, 24 loci VNTR typing and RFLP typing had similar high-level of discrimination among 95 Beijing strains from Southern Vietnam. However, loci VNTR 154, VNTR 2461 and VNTR 3171 had hardly added any value to the level of discrimination.

\section{Background}

The IS6110 restriction fragment length polymorphism (RFLP) typing was previously considered the gold standard in the molecular epidemiology of tuberculosis [1]. Although this typing technique generally revealed a high level of discrimination among Mycobacterium tuberculosis isolates, it was considered complicated, technically

\footnotetext{
* Correspondence: maihuyen1967@yahoo.com

${ }^{\dagger}$ Equal contributors

'Phạm Ngọc Thạch hospital, 120 Hung Vuong, district 5, Ho Chi Minh City, Viet Nam

Full list of author information is available at the end of the article
}

demanding, and time consuming. In addition, a part of the strains contained too few copies of IS6110 to enable a reliable typing. Variable number of tandem repeat (VNTR) typing is easier and faster to perform, and yields results in a numerical format. Therefore, this technique has become the new international typing method for $M$. tuberculosis since 2006 [2]. Several studies indicated that VNTR typing is as discriminative as RFLP typing and more suitable to type strains with few copies of IS6110 [3,4]. However, doubt remained whether VNTR typing is as good as RFLP typing in discriminating Beijing genotype strains. As in 
Vietnam about $40 \%$ of the M. tuberculosis isolates are of this genotype, we in this study compared the performance of RFLP and internationally standardized 24 loci VNTR typing to discriminate among one hundred Beijing genotype isolates from the South of Vietnam.

\section{Methods}

\section{Study population}

In total $100 \mathrm{M}$. tuberculosis isolates of the Beijing genotype family were selected from a previous study on the dynamics of tuberculosis transmission in Vietnam. The study area consisted of three adjacent rural districts in Tiengiang Province, in the Mekong River Delta in Southern Vietnam. All patients were aged $\geq 15$ years, resident in the study area and registered for treatment of smear-positive pulmonary tuberculosis (TB) between 1 January 2003 and 28 June 2007 at the participating District Tuberculosis Units, or at the provincial TB hospital and were eligible for inclusion into the study. Each eligible patient submitted two sputum samples for TB culture, drug susceptibility testing and genotyping and completed an interview form. The details of this study have been published previously [5].

\section{Ethical approval}

Ethical clearance was obtained from the ethical health committee of the Ho Chi Minh City Council (reference number 1106/UBND-VX). All included patients provided written informed consent.

\section{Mycobacterium tuberculosis culture}

Sputum specimens were kept refrigerated and transported to Pham Ngoc Thach Hospital in Ho Chi Minh City within $72 \mathrm{hrs}$ after collection. They were decontaminated and liquefied using $1 \% \mathrm{~N}$-acetylcysteine $/ 2 \% \mathrm{NaOH}$, inoculated on modified Ogawa medium and incubated at $37^{\circ} \mathrm{C}$. Cultures were examined for growth after 1, 2, 4, 6 and 8 weeks of incubation. Cultures with no growth after 8 weeks were considered negative. M. tuberculosis was identified using the niacin and the nitrate tests [6].

\section{DNA typing}

Genomic DNA was extracted from positive cultures using an earlier described method [7]. IS6110 RFLP typing and spoligotyping were performed according to the internationally standardized methods $[1,8]$. The VNTR typing was executed on the basis of 15 loci and 24 loci as described by Supply et al. [2].

\section{Random selection of one hundred Beijing genotype strains}

Among 1,797 M. tuberculosis isolates that were successfully typed in RFLP and spoligotyping, 819 strains represented the Beijing genotype according to spoligo typing patterns. After the isolate numbers had been sorted in numerical order, every $8^{\text {th }}$ Beijing genotype was selected until a total of 100 isolates was reached.

\section{Data analysis}

Gene Marker software, version 1.5 (Softgenetics, PA, USA) was used for analysis and automated allele calling of the VNTR patterns. The Bionumerics software, version 3.0 (Applied Maths, Sint-Martens Latem, Belgium) was used for the analysis and comparison of IS6110 RFLP and VNTR typing patterns.

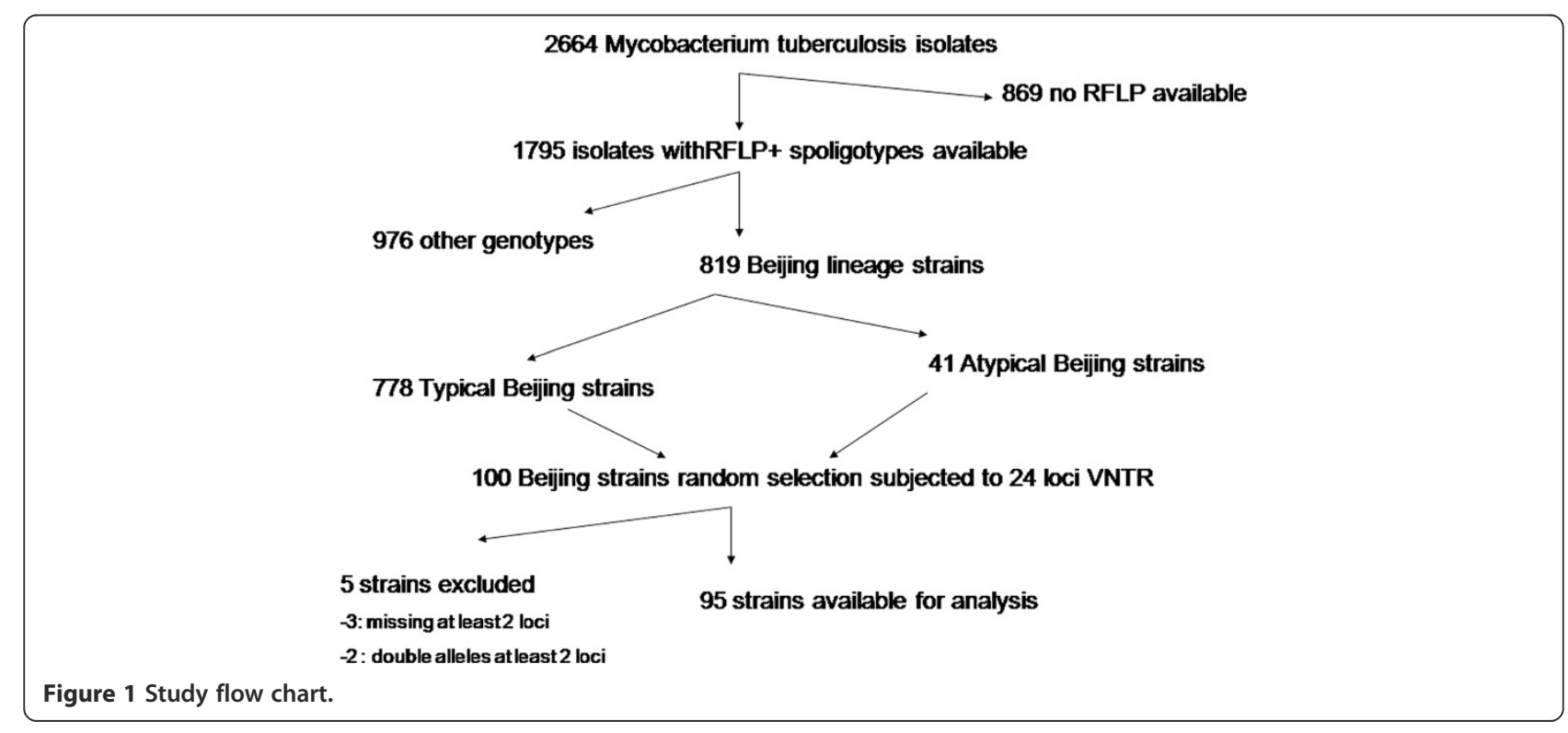


The Hunter Gaston Discrimination Index (HGDI) was used to analyse the discrimination power of VNTR and RFLP typing results [9]:

$$
D=1-\frac{1}{n(n-1)} \sum_{j=1}^{S} n j(n j-1)
$$

where $\mathrm{n}$ is the total number of strains in the sample population, $\mathrm{s}$ is the total number of types described, and $\mathrm{nj}$ is the number of strains belonging to the $\mathrm{j}^{\text {th }}$ type. This equation is derived as follows: the probability that a single strain sampled at random will belong to the $\mathrm{j}^{\text {th }}$ group is $\mathrm{nj} / \mathrm{n}$ and the probability that two strains sampled consecutively will belong to that group is $n j(n j-1) / n(n-1)$.

\section{Definitions}

Beijing lineage (genotype) strains were defined as strains having at least three of the nine spacers 35 to 43 and lacking spacers $1-34$ based on the 43 spacer spoligo patterns $[8,10]$. If a strain missed all spacers $1-34$ and also one or a few of the spacers 35-43, the Beijing strains was considered to represent the Atypical branch of the Beijing genotype lineage [11].

Two strains were defined as a cluster if they had identical RFLP patterns or identical VNTR profiles (Bionumerics), or if VNTR types differed by no more than a single locus [2].

\section{Results}

In the period January 2003 to June 2007, a total of 2,664 M. tuberculosis strains were isolated from eligible patients, of which 1,795 were successfully typed in RFLP and spoligo typing. Of these, 819 (45.6\%) were of the Beijing genotype based on the spoligo patterns; the remaining 976 (54.4\%) were of other genotypes. Among the 819 Beijing genotype strains, 41 (5.0\%) most likely belonged to Atypical Beijing lineage, as they missed one or more spacers of the characteristic 9 spacer signature.

Of the 819 Beijing strains, 353 (43.1\%) were isolated from patients in the Cailay district; 221 strains (27\%) isolated from patients in the Caibe district, and the remaining 245 strains (29.9\%) from patients in the Chauthanh district. Regarding the gender of patients, $592 / 819(72.3 \%)$ of the strains were isolated from male patients, the remaining 227 (27.7\%) from females.

From these 819 Beijing genotype strains, 100 strains were randomly selected as described above, of which $5 \%$ were most likely Atypical Beijing strains because they missed one or two spacers of the characteristic nine spacer panel 35-43.

Among the 100 selected Beijing genotype strains, 38 (38\%) were isolated in the Cailay district, 32 (32\%) in the Caibe district and 30 (30\%) in the Chauthanh district. The gender distribution was $71 \%$ males and $29 \%$ female patients. Therefore, the distribution of sex, districts, age (data not shown) in the representative Beijing strains was similar to that in the total collection of 819 Beijing strains $(\mathrm{P}>0.05)$.

Among the 100 Beijing isolates that were subjected to 24 loci VNTR typing, 95 yielded results suitable for analysis (including 88 that yielded results for all 24 loci and 7 with double alleles or that missed one locus only), the remaining 5 strains were excluded because ambiguous PCR results were obtained or double alleles were observed in at least two loci. Among 3/5 isolates at least two loci could not be amplified and two isolates had double alleles in two or more loci (Figure 1).

Of the 24 loci analyzed, VNTR 154, VNTR 2461 and VNTR 3171 had no discrimination power and hence, the HGDI was zero. Loci VNTR 2347, VNTR 580, VNTR1644, VNTR 0577, VNTR 2531, VNTR 2401 and VNTR 802 had a HGDI of less than 0.2. The loci having a HGDI of more than 0.4 were VNTR 424, VNTR 960, VNTR 2996, VNTR 4052, VNTR1955, VNTR 2165 and

\section{Table 1 Hunter Gaston discrimination index (HGDI) values obtained for each locus in the present study compared to two other studies}

\begin{tabular}{|c|c|c|c|}
\hline VNTR locus & Present study & Kremer et al. [4] & Alonso et al. [12] \\
\hline 154 & 0.00 & & 0.23 \\
\hline 2461 & 0.00 & 0.00 & \\
\hline 3171 & 0.00 & & \\
\hline 2347 & 0.02 & & \\
\hline 580 & 0.02 & 0.019 & 0.21 \\
\hline 1644 & 0.06 & 0.058 & 0.455 \\
\hline 577 & 0.06 & 0.165 & 0.63 \\
\hline 2531 & 0.08 & & 0.655 \\
\hline 2401 & 0.10 & & 0.65 \\
\hline 802 & 0.16 & 0.196 & 0.73 \\
\hline 3192 & 0.21 & & 0.36 \\
\hline 4156 & 0.23 & & 0.53 \\
\hline 2687 & 0.24 & & 0.06 \\
\hline 2059 & 0.26 & & 0.16 \\
\hline 3690 & 0.27 & & 0.64 \\
\hline 3007 & 0.31 & & 0.13 \\
\hline 4348 & 0.37 & 0.32 & 0.09 \\
\hline 424 & 0.42 & & 0.66 \\
\hline 960 & 0.44 & 0.377 & 0.685 \\
\hline 2996 & 0.46 & 0.2 & 0.46 \\
\hline 4052 & 0.50 & 0.299 & 0.8 \\
\hline 1955 & 0.57 & & 0.65 \\
\hline 2165 & 0.635 & 0.201 & 0.61 \\
\hline $2163 b$ & 0.64 & 0.618 & 0.78 \\
\hline
\end{tabular}


VNTR 2163b. Locus VNTR 2163b had the highest allelic diversity, with a HGDI of 0.64 (Figure 2 and Table 1).

In the 24 loci VNTR typing of the remaining 95 isolates, 14 clusters of isolates were found; one cluster of four isolates, four clusters of three isolates and nine clusters of two strains. Sixty-one isolates revealed a unique VNTR pattern. The HGDI amounted to 0.994. Twelve of the VNTR clusters were subdivided in RFLP typing.

In RFLP typing, 13 clusters of isolates were found, comprising in total of 31 strains. There were ten clusters of two, two clusters of three and one cluster of five isolates. Sixty-four isolates had a unique RFLP pattern $($ HGDI $=0.994)$. Nine RFLP clusters were subdivided in VNTR typing.

\section{Discussion}

From 1993 to 2006, RFLP typing was considered the gold standard in typing of $M$. tuberculosis isolates, especially for strains harboring multiple IS6110 copies, like the ones of the Beijing genotype family. However, this typing method is technically demanding and time consuming [4]. Furthermore, the discriminatory power of RFLP typing among strains with a low number of IS6110 copies ( $<=5$ copies $)$ is very poor [3]. Therefore, in recent years VNTR typing has increasingly been explored in the molecular epidemiology worldwide and with the proposal on international standardization of this technique in 2006 it has in fact become the new gold standard [2]. VNTR typing introduced major advantages in typing in comparison to RFLP typing, such as its ease in use, its suitability for standardization, and that the results that are displayed in numbers can be analyzed easily and exchanged efficiently between laboratories. Moreover, the turnaround time of VNTR typing is much shorter than that of RFLP typing, because it is PCRbased and only a little amount of mycobacterial DNA is required.

Many researchers have carried out comparisons between RFLP and 12 or 15 loci VNTR typing methods for discriminating $M$. tuberculosis isolates [3,4,12]. Their findings showed that the discriminative power of 12 loci VNTR was lower than that of 15 loci VNTR (with HGDI of 0.978-0.995) [12] and 15 loci VNTR has high level of discrimination with HGDI 0.990-0.995 [4,12], but this was still lower than that of RFLP typing (0.998) [4]. However, Supply et al. [2] proposed to apply 24 instead of 15 loci in VNTR typing, and this improved the level of discrimination significantly.

In our study, we compared the performance of 15 and 24 loci VNTR typing and RFLP typing using 95 Beijing strains and we found that the discrimination index (HGDI) of 15 loci VNTR was the lowest (0.992), followed by both RFLP typing and 24 loci VNTR typing

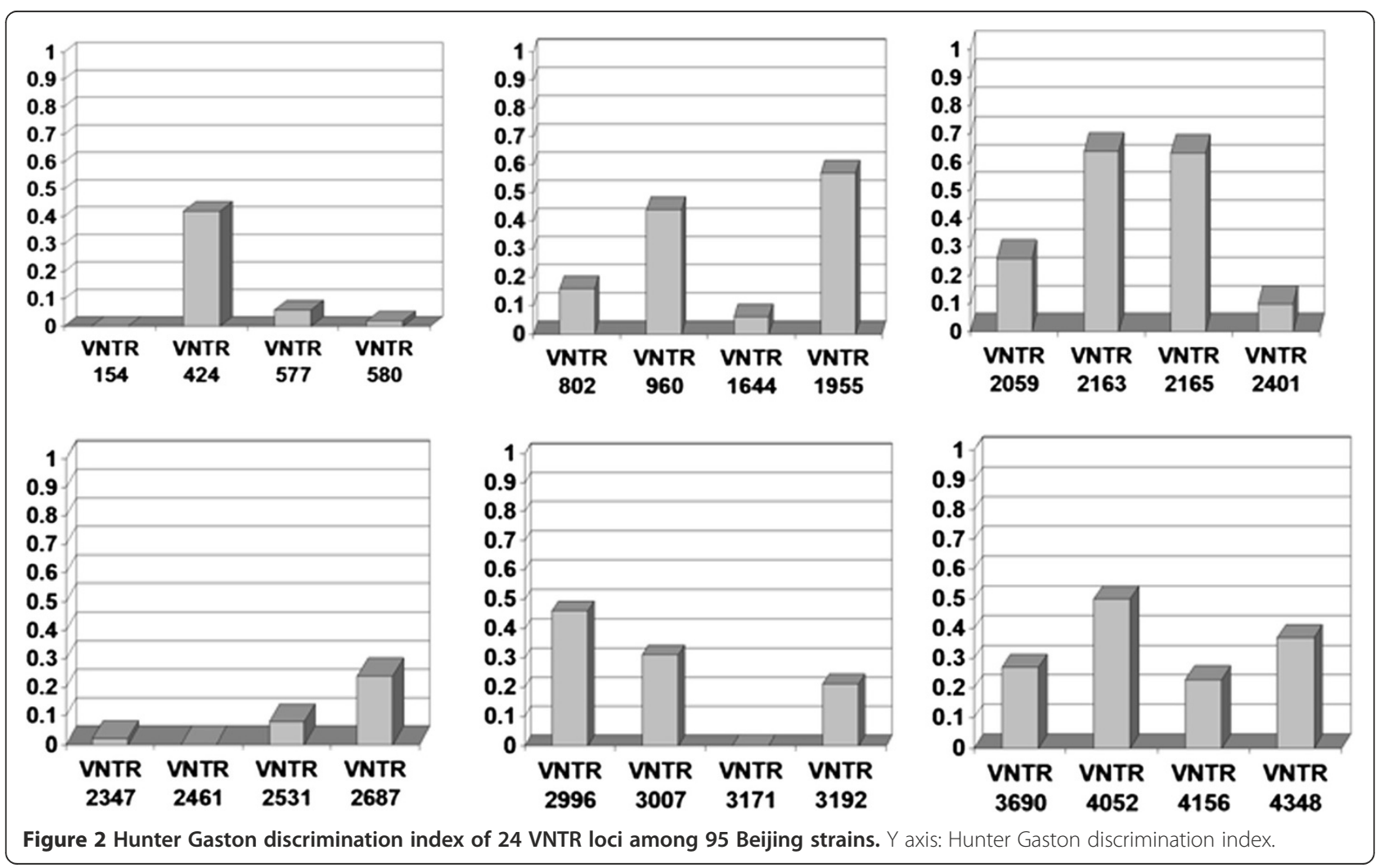


(0.994). However, the differences observed were small. The HGDI of some loci (VNTR 154, VNTR 2461,VNTR 3171) were low in our study, which means that these loci are less useful in discriminating Beijing strains in the South of Vietnam and presumably elsewhere. The HGDI of VNTR 2461, VNTR 577, VNTR 2163b, VNTR 580, VNTR 802, VNTR 960, VNTR 1644 and VNTR 4348, were similar to that observed in a previous study in Hong Kong [4] (Table 1), whereas the HGDI of VNTR 2996, VNTR 4052, and VNTR 2165 were significantly higher than the ones in that study [4] (Table 1), for unknown reasons. It may be that because BCG vaccination has been introduced much earlier in Hong Kong than in Vietnam, the ongoing selection of particular strains of the Beijing lineage [11] may be more advanced in the former than in the latter area and the mentioned loci may have a different level of discriminative power among the circulating strains in both areas.

Our study further found the HGDI of VNTR 1955, $2163 \mathrm{~b}$ and 2165 to be very high $(>0.50)$ and the best differentiation, similar to two previous studies $[4,12]$, was obtained with VNTR 2163b (Table 1 and Figure 2).

Some of the HGDI of individual loci in our study were significantly different to the ones found in the study of Alonso et al. [12] (Table 1), because we performed VNTR typing of exclusively Beijing strains, whereas Alonso et al. [12] carried out VNTR typing on a strain collection consisting of 32\% LAM, 28\% Haarlem and only $2 \%$ Beijing strains.

A disadvantage of VNTR typing encountered in this study was that six strains revealed double alleles in a single locus, and two strains even in two and more than two loci. It is not clear whether the latter observation was associated with a mixed infection [13]. However, the revealed genomic instability in particular loci decreases the utility of VNTR typing significantly, as this hampers a reliable interpretation. Also in RFLP typing transposition of IS6110 sometimes interfered with a reliable interpretation, but such a genetic turn-over was observed less frequently [14]. However, we cannot exclude the possibility that these multiple alleles may reflect important phenomena in the epidemiology of TB currently unknown, and these observations, although technically demanding, may be associated with the ongoing adaptation of $M$. tuberculosis to the current TB control measures.

A major limitation of this study was that we did not have epidemiological information available to verify the transmission links indicated by both typing methods. It was therefore, not possible to ascertain the validity of epidemiological links indicated.

\section{Conclusions}

In comparison to 15 loci VNTR, RFLP typing and 24 loci VNTR typing revealed the highest level of discri- mination among 95 isolates of the Beijing genotype from Southern Vietnam. For this and other practical reasons, the last method is preferred in investigation on transmission of Beijing strains in Vietnam. This method is in principle also useful in screening for possible mixed infections, after which positive findings (more than two loci with double alleles) would be confirmed with other methods.

\section{Competing interests}

The authors declare that they have no competing interests.

\section{Authors' contributions}

$\mathrm{MH}$ was involved in data collection, analysis and in writing the manuscript. $\mathrm{KK}, \mathrm{NL}, \mathrm{ET}, \mathrm{FC}, \mathrm{PH}$ and DS were involved in the conception of the study and in writing the manuscript. TB was involved in data collection and in writing the manuscript. All authors read and approved the final manuscript.

\section{Acknowledgements}

This research was funded by the KNCV Tuberculosis Foundation, the Medical Committee Netherlands-Vietnam, the Netherlands.

We thank all TB patients participating in this study and the staff of the National Tuberculosis Program of Tien Giang province for recruiting the patients as well as the staff of the National Tuberculosis Program in PNT hospital for delivering, supervising and checking the data. DaiViet Hoa, Phan Thi Hoang Anh and other staff in Pham Ngoc Thach laboratory are deeply thanked for performing culture, DST and GenoType ${ }^{\circledR}$ MTBDRplus testing. We are grateful to Anne-Marie van den Brandt, Mirjam Dessens, Mimount Enaimi and Arnout Mulder, Jessica de Beer and other staff of the Tuberculosis Reference Laboratory at the National Institute for Public health and the Environment (The Netherlands) for providing us the knowledge and practice on the molecular techniques used in this study.

\section{Author details}

1Phạm Ngọc Thạch hospital, 120 Hung Vuong, district 5, Ho Chi Minh City, Viet Nam. ${ }^{2}$ World Health Organization, Regional Office for Europe, Copenhagen, Denmark. ${ }^{3}$ Tuberculosis Reference Laboratory, RIVM, PO Box 1, Bilthoven 3720 BA, The Netherlands. ${ }^{4}$ Centre for Infection and Immunity Amsterdam, Academic Medical Centre, Amsterdam, The Netherlands. ${ }^{5}$ KNCV Tuberculosis Foundation, PO Box 146, The Hague 2501 CC, The Netherlands. ${ }^{6}$ Departments of Pulmonary Diseases and Medical Microbiology, Radboud University, PO box 9101, Nijmegen 6500 HB, The Netherlands.

Received: 23 July 2012 Accepted: 29 January 2013

Published: 2 February 2013

\section{References}

1. van Embden JD, Cave MD, Crawford JT, Dale JW, Eisenach KD, Gicquel B, Hermans P, Martin C, McAdam R, Shinnick TM, et al: Strain identification of Mycobacterium tuberculosis by DNA fingerprinting: recommendations for a standardized methodology. J Clin Microbiol 1993, 31:406-409.

2. Supply P, Allix C, Lesjean S, Cardoso-Oelemann M, Rusch-Gerdes S, Willery E, Savine E, de Haas P, van Deutekom H, Roring S, et al: Proposal for standardization of optimized mycobacterial interspersed repetitive unitvariable-number tandem repeat typing of Mycobacterium tuberculosis. J Clin Microbiol 2006, 44:4498-4510.

3. Smittipat $N$, Billamas $P$, Palittapongarnpim $M$, Thong-On A, Temu MM Thanakijcharoen P, Karnkawinpong O, Palittapongarnpim P: Polymorphism of variable-number tandem repeats at multiple loci in Mycobacterium tuberculosis. J Clin Microbiol 2005, 43:5034-5043.

4. Kremer K, Au BK, Yip PC, Skuce R, Supply P, Kam KM, van Soolingen D: Use of variable-number tandem-repeat typing to differentiate Mycobacterium tuberculosis Beijing family isolates from Hong Kong and comparison with IS6110 restriction fragment length polymorphism typing and spoligotyping. J Clin Microbiol 2005, 43:314-320.

5. Buu TN, Huyen MN, Lan NT, Quy HT, Hen NV, Zignol M, Borgdorff MW Cobelens FG, van Soolingen D: The Beijing genotype is associated with young age and multidrug-resistant tuberculosis in rural Vietnam. Int $\mathrm{J}$ Tuberc Lung Dis 2009, 13:900-906 
6. International Union Against Tuberculosis and Lung Disease: Technical guidesputum examination for tuberculosis direct microscopy in low-income countries. In The Union. 5th edition. Paris, France; 2000.

7. Van Soolingen D, de Haas PEW, Kremer K: Restriction fragment length polymorphism typing of mycobacteria. In Mycobacterium tuberculosis protocols. Edited by Parish T, Stoker NG. Totowa, New Jersey, USA: Humana Press Inc; 2001:165-203.

8. Kamerbeek J, Schouls L, Kolk A, van Agterveld M, van Soolingen D, Kuijper S, Bunschoten A, Molhuizen H, Shaw R, Goyal M, et al: Simultaneous detection and strain differentiation of Mycobacterium tuberculosis for diagnosis and epidemiology. J Clin Microbiol 1997, 35:907-914.

9. Hunter PR, Gaston MA: Numerical index of the discriminatory ability of typing systems: an application of Simpson's index of diversity. J Clin Microbiol 1988, 26:2465-2466.

10. Kremer K, Glynn JR, Lillebaek T, Niemann S, Kurepina NE, Kreiswirth BN, Bifani PJ, van Soolingen D: Definition of the Beijing/W lineage of Mycobacterium tuberculosis on the basis of genetic markers. J Clin Microbiol 2004, 42:4040-4049.

11. Kremer K, Van der Werf MJ, Au BK, Anh DD, Kam KM, Van Doorn HR, Borgdorff MW, Van Soolingen D: Vaccine-induced immunity circumvented by typical Mycobacterium tuberculosis Beijing strains. Emerg Infect Dis 2009, 15:335-339.

12. Alonso Rodriguez N, Martinez Lirola M, Herranz M, Sanchez Benitez M, Barroso P, Bouza E, Garcia De Viedma D: Evaluation of the new advanced 15-loci MIRU-VNTR genotyping tool in Mycobacterium tuberculosis molecular epidemiology studies. BMC Microbiol 2008, 8:34.

13. Shamputa IC, Jugheli L, Sadradze N, Willery E, Portaels F, Supply P, Rigouts $L$ : Mixed infection and clonal representativeness of a single sputum sample in tuberculosis patients from a penitentiary hospital in Georgia. Respir Res 2006, 7:99.

14. de Boer AS, Borgdorff MW, de Haas PE, Nagelkerke NJ, van Embden JD, van Soolingen D: Analysis of rate of change of IS6110 RFLP patterns of Mycobacterium tuberculosis based on serial patient isolates. I Infect Dis 1999, 180:1238-1244.

doi:10.1186/1471-2334-13-63

Cite this article as: Huyen et al:: Clustering of Beijing genotype

Mycobacterium tuberculosis isolates from the Mekong delta in Vietnam on the basis of variable number of tandem repeat versus restriction fragment length polymorphism typing. BMC Infectious Diseases 2013 13:63.

\section{Submit your next manuscript to BioMed Central and take full advantage of:}

- Convenient online submission

- Thorough peer review

- No space constraints or color figure charges

- Immediate publication on acceptance

- Inclusion in PubMed, CAS, Scopus and Google Scholar

- Research which is freely available for redistribution 